\title{
Network Structure and Topology based Routing Techniques in Wireless Sensor Network- A survey
}

\author{
Rajendra Singh Bisht \\ M.Tech Final Year \\ Computer Science \& Engineering \\ BTKIT Dwarahat, Uttarakhand, India
}

\author{
Shailendra Mishra,Ph.D \\ Head of Department \\ Computer Science \& Engineering \\ BTKIT Dwarahat, Uttarakhand, India
}

\begin{abstract}
Routing techniques in Wireless Sensor Network has always been important area of exploration due to change in demands of various applications such as Area monitoring, Environmental/Earth monitoring, passive localization and tracking, Industrial monitoring etc. Energy efficiency is crucial issue in WSN because sensor nodes in WSN are with limited battery power/Resources . This results in rapid change in design of routing algorithms. Day by day various routing techniques are being proposed. It is because of energy efficiency, Scalability, Network lifetime and other important factors of routing techniques. Each routing mechanism has its advantages and disadvantages related to energy efficiency also. There is no single, best routing protocol that is suitable for all applications. Routing mechanism might differ depending on the application, network architecture and topologies. This paper gives review of recent research of the network structure and topology based different routing strategies and gives brief idea about energy efficiency of routing protocols in WSN. This paper also represents classification of various routing techniques with their advantages and disadvantages.
\end{abstract}

\section{Keywords}

Base station (sink), Clustering, Energy efficiency, Routing Protocols, Wireless Sensor Network

\section{INTRODUCTION}

A Wireless Sensor Network (WSN) consists of various tiny nodes (called sensors) densely deployed in a small or large geographical area to monitor physical or environmental conditions, such as sound, temperature, Air direction, pressure, etc. wireless sensor networks are broadly used in civil, military ,security applications. A sensor has some capabilities which include collection/gathering of data from physical geographical area using embedded micro processor, aggregation of data, receiving of data, sending of data using radio transmitter to another similar node or to a sink (also called base station). This all work carried out by a node require energy to consume but nodes in WSN have limited battery power. Therefore, energy efficiency has always been a crucial factor and a challenge in the design of routing algorithms. Along with this, nodes in WSN have limited memory, limited computational capabilities. These nodes also can be considered as a collection of low-cost, low-power, and multifunctional wireless sensor nodes. WSNs are different from traditional wireless communication system (such as cellular systems and mobile ad hoc networks). Design issues for both systems are also different in nature. While designing routing protocols for WSNs the energy efficiency factor, fault tolerance, scalability of these protocols should be considered. To replace these challenges with proper solution many routing techniques have already been proposed in area of WSN. This paper describes existing routing techniques based on network structure and topologies in WSN and also present recent research of routing techniques for wireless sensor networks, including their advantages and disadvantages.

\section{ROUTING TECHNIQUE AND ITS DESIGN CONSIDERATION IN WSN}

Routing is a technique of determining path from source to destination upon request of data transmission. Routing table is used to maintain address of nearest node. There may be one or more intermediate node/s between source and destination. A routing algorithm performs routing and maintenance of routing table.

While designing the routing algorithms following consideration must be taken into account [1]

1. Sensor nodes in WSN may be stationary or mobile.

2. Single-hop routing technique consumes more energy than multi-hop but multi-hop results in overhead for topology management and medium access control.

3. Data delivery model (Reliability) to the sink can be continuous, event driven, query-driven and hybrid, depending on the application of the sensor network.

4. Data can be aggregated to remove redundancy.

5. Sensor node deployment is either deterministic or selforganizing.

6. A sensor node in WSN is multifunctional. It collects data, aggregates data, transmits data, and routes the data.

7. Power failure, physical damage or environmental interferences are cause of sensor node failure.

The objectives of routing technique should be correct delivery of data (Real or non-real time) and maximization of network life time.

\section{CLASSIFICATION OF ROUTING TECHNIQUES}

Many routing techniques have already been proposed in the area of wireless sensor network. Routing algorithms/Techniques have been classified in a number of ways by various researchers. There is no general classification is available till today. Some researchers [5, 22, and 23] have classified routing algorithms into three broad categories Data-centric Routing Protocols, Hierarchical Routing Protocols and Location-based Routing Protocols. Here a more general classification of routing techniques is presented. Most of the routing techniques fall into one of the following category.

Routing techniques can be classified based on following:

- On the basis of Route Selection or path establishment [4, $5,6,7,24]$

1. Proactive protocols

2. Reactive protocols

3. Hybrid protocols

- On the basis of protocol Operation [4, 6,24]

1. Negotiation based routing

2. Multipath routing protocols 
3. Query based routing

4. QOS based routing

5. Coherent routing

- On the basis of Number of path [4]

1. Uni-path

2. Multi-path

- On the basis of Network structure, topology and Geographical position information [4, 6,24 ]

1. Data centric or Flat

2. Hierarchical

3. Location-based

The main focus of this paper is on routing techniques that are based on network structure, topology and Geographical position information.

\section{DATA-CENTRIC OR FLAT ROUTING}

In WSNs due to large number of sensor nodes deployed it is not possible to uniquely find every node. So data centric approach is used to address this problem. In this technique certain regions are selected by the base station (sink) for query and sensor in selected region reply with data. The naming should be based on attribute since data is being requested through queries. This attribute based naming specifies the properties of data.

Examples of Data-centric based routing protocol

4.1 DD-Directed Diffusion

4.2 Flooding and Gossiping

4.3 SPIN-Sensor Protocols for Information Negotiation

4.4 Rumor routing

\subsection{Directed Diffusion}

In Directed diffusion [8] attribute-value pairs for the data is used that ensure on-demand mechanism. In the first step interest propagation is done by Base station (Or Sink) to or through its neighbors. In the next step source sends a gradient (characterized by the data rate, delay time and expiration time) as a reply link to neighbor nodes from which interest was received. In last step these interest and gradient are used to establish a path between source and sink. There is probability of establishment of path more than one so one path is selected by reinforcement using gradient factor. When the source sensor node responds to the BS there may be multiple routes from where BS receives data. Then base station has to select the gradient having minimum delay time. DD is an on-demand routing mechanism. As compare to other technique its ondemand mechanism saves lot of energy. Figure 1 shows Working of DD routing technique.

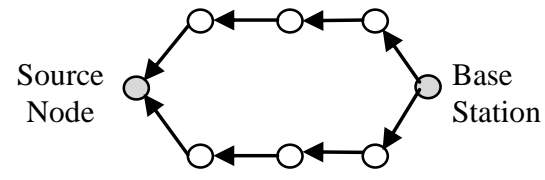

(A)

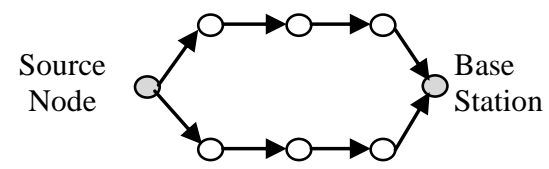

(B)

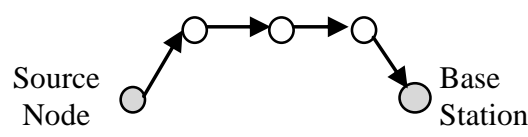

$\mathrm{O} 0 \mathrm{O}$

(C)

Fig. 1: Working of Directed diffusion routing technique (A) Interest Propagation, (B) Send Gradients, (C) Path Establishment and Data Transmission

\subsection{Flooding and Gossiping}

Flooding [9] works as its name implies. As node receives data it broadcast it to all neighbor nodes. This happen again and again until data arrives at destination node or maximum number of hops is reached. Flooding has several drawbacks such as duplicate data sent to same node; two nodes may sense same data and send it to same neighbor.

In gossiping instead of broadcasting data it is send to randomly selected nodes. This avoids the problem of Implosion. Time taken to propagate message is very long in this scheme. It has less overhead than Flooding. It does not guarantee that all nodes of the Network will receive the message.

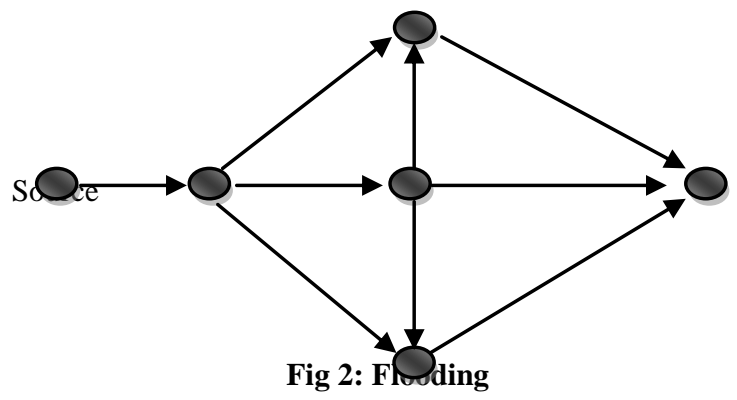

\subsection{Spin-Sensor Protocols for Information via Negotiation}

In SPIN [10] data advertisement mechanism i.e. key feature of this technique is used. In this scheme three messages are used in sequence. As a new data arrives at node, the node send a advertise message (first message i.e. advertise) to its neighbors. Now only those neighbors request (second message i.e. request) for the data that do not have the same data and finally data is sent to the requested nodes (third message i.e. data). It overcomes the problem of redundant data passing that was the drawback of flooding technique. One of the advantages of SPIN is that topological changes are localized since every node needs to know its neighbors only that can be reached using single hop. Figure 3 shows the working of SPIN routing protocol. 


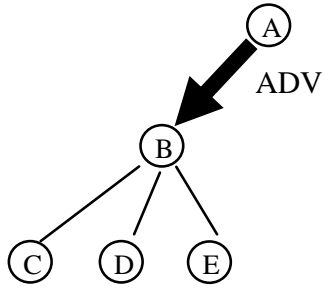

(a)

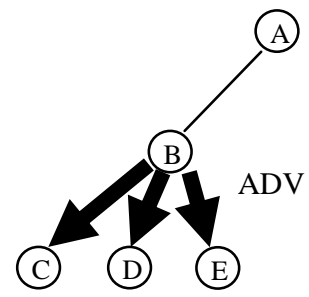

(d)

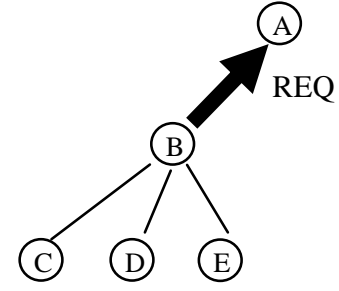

(b)

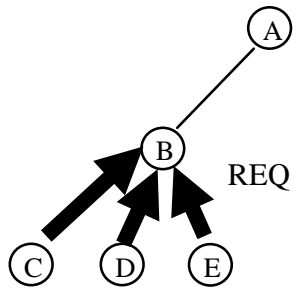

(e)

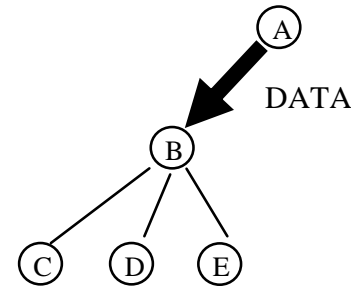

(c)

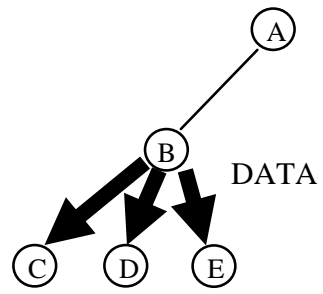

(f)

Fig. 3: S PIN-node A advertise its data to node B (a), node B responds by sending a request to node A (b), node A sends data to node $B$ (c), node $B$ then sends advertisements to its neighbors (d), who in turn send requests back to $B$ (e-f)

But in terms of reliability SPIN does not guarantee the delivery of data e.g. if a node that is far away from source node is interested in data and intermediate nodes are not interested in that data then such data will not be delivered to requested node. This can be considered as drawback of SPIN because it cannot be used in application where delivery of data is primary requirement of application. As compare to directed diffusion it does not have on-demand mechanism.

\subsection{Rumor Routing}

Rumor routing [11] is similar to Directed Diffusion technique and used in application where Geographical routing fails. In this technique agents (long-lived packets) are used to flood event. Nodes maintain an event table. As nodes detect an event it updates event table and create an agent. Agent propagates information by travelling in the network. A query is generated for an event is responded by nodes that know the route. Event table is used for this purpose. Energy is saved by reducing communication cost because there is no need of flooding information on the entire network. RR maintains only single path between source and sink.

\section{HIERARCHICAL ROUTING}

Hierarchical Routing techniques are well known for its scalability and energy efficiency. Algorithm under this category sometimes classified as energy-efficiency routing algorithms. These techniques are also called clustering techniques because nodes within a WSN are divided into different clusters. That is why these techniques are known as

"ENERGY-EFFICIENT HIERARCHICAL CLUSTER ROUTING ALGORITHMS". Nodes with higher energy are used to process and transmission data while nodes with less energy are used to sense and collect data. Cluster formation, Cluster head selection and rotation are major activities of this technique that results in maximization of network life time.
Multi-hop communication is also employed by this technique to save energy consumption.

Examples of Hierarchical Routing based routing protocol

5.1 LEACH

5.2 PEGASIS

5.3 HEED

5.4 TEEN

5.5 APTEEN

\subsection{LEACH}

Low-energy adaptive clustering hierarchy (LEACH) [2] is very widely known algorithm. In this technique wireless sensor nodes form clusters on the basis of energy stored. Each cluster has its cluster head $(\mathrm{CH})$ that takes the duty of data transmissions while other nodes of that cluster sense and collect the data. Data fusion and aggregation are local to the cluster. A $\mathrm{CH}$ can communicate with other $\mathrm{CH}$ or with Base station (Sink).To balance the energy dissipation of node Cluster head rotation is performed after specified round of communication. The overall working of LEACH can be classified in to two phase first is setup phase and the second is steady state phase. Setup phase includes cluster formation and $\mathrm{CH}$ selection while in steady state phase data is transmitted to sink.

A sensor node selects a number $\mathrm{r}$ randomly that must lie between 0 and 1 . If this $r$ is less than $T(n)$ i.e. threshold value, the Node becomes a cluster-head for the current round.

Following equation is used to calculate threshold value:

$$
\mathrm{T}(\mathrm{n})=\left\{\begin{array}{cl}
\frac{\mathrm{p}}{1-\mathrm{p}(\mathrm{r} \bmod (1-\mathrm{p}))} & \text { if } \mathrm{n} \in \mathrm{G} \\
0 & \text { otherwise }
\end{array}\right.
$$


$\mathrm{G}$ is the set of nodes involved in the $\mathrm{CH}$ election. After becoming $\mathrm{CH}$ each elected $\mathrm{CH}$ broadcast message to the all other nodes that they have become the new $\mathrm{CH}$.

During steady state phase Data aggregation is done by cluster head before sending the data to base station. Then again network goes into setup phase to select ne $\mathrm{CH}$. To reduce interference different clusters use different codes to communicate.

\subsection{PEGASIS}

Power-Efficient Gathering in Sensor Information Systems (PEGASIS) [12] is a near optimal chain-based power efficient protocol. The chain is constructed in a greedy way. PEGASIS forms a chain of nodes where each node transfers and receives data from a neighbor. To locate the nearest neighbor in PEGASIS, signal strength is used to calculate distance to all neighboring nodes. In PEGASIS every node can communicate with Base station. Data is sent to the sink from nodes in chain on one node at a time basis. Data is aggregated when data move node by node. PEGASIS performs better than LEACH because of less overhead that were found in LEACH in the form of dynamic cluster formation, much number of transmissions.

\subsection{HEED}

Hybrid Energy-Efficient Distributed [13,14] has a advantage over LEACH of non -random selection of Cluster head $(\mathrm{CH})$.since in worst case distribution of $\mathrm{CHs}$ may not be even that will affect the data gathering. In HEED CH selection based on residual energy and intra-cluster communication cost as a function of cluster density or node degree i.e. number of neighbors. HEED supports heterogeneous sensor nodes.

It consists of three phases. During First phase i.e. Initialization phase the initial $\mathrm{CHs}$ nodes percentage $\mathrm{C}_{\text {prob }}$ is given to the nodes. Probability to become cluster head i.e. $\mathrm{CH}_{\text {prob }}$ is determined on the basis of $\mathrm{C}_{\text {prob}}$, estimated current residual energy i.e. $\mathrm{E}_{\text {residual }}$, Maximum Battery Energy i.e. $\mathrm{E}_{\mathrm{Max}}$

Below formula is given to calculate $\mathrm{CH}_{\text {prob }}$ :

$\mathrm{CH}_{\text {prob }}=\mathrm{C}_{\text {prob }} * \frac{\mathrm{E}_{\text {residual }}}{\mathrm{E}_{\text {Max }}}$

$\mathrm{E}_{\mathrm{Max}}$ may vary in HEED since it supports heterogeneous sensor nodes.

Second phase i.e. Repetition phase is iterated until the $\mathrm{CH}$ node was found with the least communication cost. If the node cannot find the appropriate $\mathrm{CH}$, then the concerned node itself was selected as the $\mathrm{CH}$. In third phase i.e. Finalization phase final $\mathrm{CH}$ node is selected.

As compare to LEACH, HEED prolongs network lifetime by distributing energy consumption, minimizes control overhead.

\subsection{TEEN}

Threshold sensitive Energy Efficient sensor Network protocol (TEEN) [15] was proposed for time specific application where sudden changes are possible such as sudden change in temperature. It is also used in application where periodic report is required. In this scheme instead of flat clustering (LEACH, PEGASIS) a multi level hierarchy is used. Nodes within a cluster report to their $\mathrm{CH}$ with collected/sensed data, now this $\mathrm{CH}$ sends aggregated data to Upper level $\mathrm{CH}$ until the data reaches the Base station. Fig. 4 redrawn from [15] shows how multilevel communication is done in TEEN. In TEEN two thresholds values are used to determine that when the sensor should report to $\mathrm{CH}$ with sensed data. After the cluster formation, the $\mathrm{CH}$ broadcasts two thresholds values shard threshold (HT) and soft threshold (ST) to nodes. AS a node senses a value at or beyond the HT, it sends the data when value of the attribute changes by an amount equal to or greater than the soft threshold. It consumes less energy because of spending much time in sensing data rather than transmitting.

The main drawback of TEEN is that, if the threshold values are not reached, the communication will never take place.

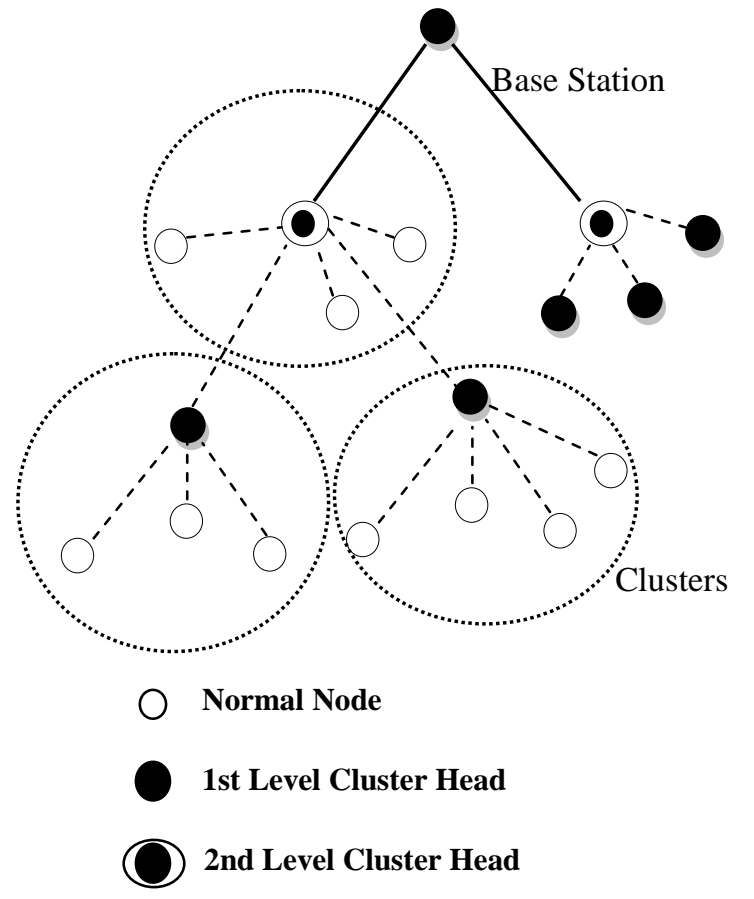

Fig. 4: Hierarchical Clustering in TEEN and APTEEN

\subsection{APTEEN}

The Adaptive Threshold sensitive Energy Efficient sensor Network protocol (APTEEN) [16] enhanced version of TEEN. In architecture APTEEN is similar to TEEN and it is hybrid clustering-based routing Scheme that allows the sensor node to send data periodically. APTEEN also react to change in the value of the sensed attribute by reporting the same values to their CHs. APTEEN guarantees lower energy consumption and longer network life time. The main drawbacks of TEEN and APTEEN are due to overhead and complexity of cluster formation because of its multi level support.

\section{LOCATION BASED ROUTING}

Location based protocols sometimes also classified as Geographic protocols. In this technique nodes are identified by its location only. Since there is no mechanism like IP address so distance between nodes is calculated using signal strength. Now this distance is used to determine energy require to transmitting the data. Nodes in this scheme sometimes are equipped with a small low power GPS receiver. Using location of sensor node query can be diffused only to that particular region where that node is present that save lots of energy by avoiding unnecessary transmissions. These techniques may be single path or multipath.

Examples of Location based routing protocols

6.1 GAF

6.2 GEAR

6.3 LEAR

6.4 MECN

6.5 SMECN 


\subsection{GAF}

In Geographic adaptive fidelity (GAF) [17] network area is divided into zones then Grid are formed. Nodes within a particular geographical region are associated with a grid. The communication cost of nodes within this grid will be same. During routing single node from grid on behalf of whole grid takes part in routing while others are in sleep mode. This saves lots of energy. As the number of nodes increases in GAF, the network lifetime is also increases.GAF is location based but may be considered as hierarchical protocol. Figure 5 redrawn from [1] show the three states involved in GAF.

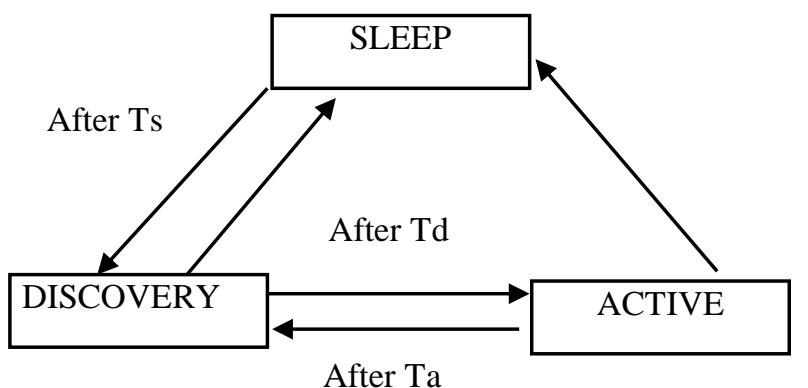

Fig. 5: Three states involved in GAF

\subsection{GEAR}

In Geographic and energy-aware routing (GEAR) [18] each node keeps an estimated cost and a learning cost of reaching the destination through its neighbors. Estimated cost includes residual energy and distance to base station both. In GEAR difference between the estimated cost and the learning cost is calculated to choose next hop. If there is no hole both cost are same but if routing lies around holes then learned cost is called refinement of the estimated cost.

There are two phases in this technique. One is the forwarding packets towards the target region; the other is the forwarding the packets within the region.

\subsection{LEAR}

Location Based Energy-Efficient Reliable Routing technique (LEAR) [19] is based on the geographic location and clustering of the nodes. A GPS device is used to compute distance from neighbors then a routing table is constructed. LEAR employ Enhanced Greedy Forwarding (EGF) algorithm that selects nearest node to the active node based on its distance.

\subsection{MECN}

In Minimum energy communication network (MECN) [20] a relay region is identified because of higher cost in direct communication. Since relay nodes are more energy efficient direct communication is avoided. Bellman-Ford shortest path is used to determine minimum energy path. MECN finds a subnetwork with less number of nodes. Global paths with minimum energy are found without considering all the nodes of Network. MECN new nodes and failure nodes doesn't affect the performance because of its self-reconfiguring mechanism.

\subsection{SMECN}

Small minimum energy communication network (SMECN) [21] is extension to MECN. The sub-network formed by SMECN for minimum energy path is smaller (in terms of number of edges) than constructed in MECN which results in decreased number of transmission hops. However SMECN has an extra overhead of finding sub-network with smaller number of edges.

\section{CONCLUSION AND OPEN ISSUES}

Routing techniques still is research area in wireless sensor network. According to various applications there are various techniques available with their features. Each Routing technique has its some advantages and also some disadvantages. There is no single, best routing protocol that is suitable for all applications. Routing mechanism might differ depending on the application, network architecture and topologies. Energy efficiency, Scalability, Fault tolerance, quality of service, Application requirements are the main challenges that play important role in evolution of routing techniques. Although many of these routing techniques look promising but still there are various open issues available and further research would be needed to address issues. In future work there may be routing techniques dealing with heterogeneous sensor nodes, Wireless mobile sensor nodes that are not supported by existing techniques.

\section{REFERENCES}

[1] Kemal Akkaya, Mohamed Younis, "A Survey on routing protocols for Wireless Sensor Networks", Ad Hoc Networks 3 (2005) 325-349.

[2] Heinzelman W, Chandrakasan A, Balakrishnan H. Energyefficient communication protocol for wireless microsensor networks. Proceedings of the 33rd International Conference on System Science (HICSS'00), Hawaii, U.S.A., January 2000.

[3] I.F. Akyildiz et al., Wireless sensor networks: a survey, Computer Networks 38 (4) (2002) 393-422.

[4] J. N. Al-Karaki and A. E. Kamal, "Routing Techniques in Wireless Sensor Networks: A Survey", IEEE Wireless Communications Magazine, vol. 11, no. 6, pp. 6-28, December, 2004.

[5] Subhadra Shaw (Bose), Energy-Efficient Routing Protocol in Wireless Sensor Network, International Journal of Scientific \& Engineering Research Volume 2, Issue 12, December-2011.

[6] Prabhat Kumar, M.P.Singh and U.S.Triar A Review of Routing Protocols in Wireless Sensor Network, International Journal of Engineering Research \& Technology (IJERT).

[7] Neha Rathi, Jyoti Saraswat and Partha Pratim Bhattacharya, A review on routing protocols for application in wireless sensor networks,International Journal of Distributed and Parallel Systems (IJDPS) Vol.3, No.5, September 2012.

[8] C. Intanagonwiwat, R. Govindan, D. Estrin, Directed diffusion: a scalable and robust communication paradigm for sensor networks in Proceedings of the 6th Annual ACM/IEEE International Conference on Mobile Computing and Networking (MobiCom_00), Boston, MA, August 2000.

[9] S. Hedetniemi, A. Liestman, A survey of gossiping and broadcasting in communication networks, Networks 18 (4) (1988) 319-349.

[10] W. Heinzelman, J. Kulik, H. Balakrishnan, Adaptive protocols for information dissemination in wireless sensor networks in Proceedings of the 5th Annual ACM/IEEE International Conference on Mobile Computing and Networking (MobiCom_99), Seattle, WA, August 1999.

[11] D. Braginsky, D. Estrin, Rumor routing algorithm for sensor networks, in: Proceedings of the First Workshop on 
Sensor Networks and Applications (WSNA), Atlanta, GA, October 2002.

[12] S. Lindsey, C.S. Raghavendra, PEGASIS: power efficient gathering in sensor information systems, in: Proceedings of the IEEE Aerospace Conference, Big Sky, Montana, March 2002.

[13] Ossama Younis and Sonia Fahmy, Distributed Clustering in Ad-hoc Sensor Networks: A Hybrid, Energy-efficient Approach, September 2002.

[14] Ossama Younis and Sonia Fahmy. Heed: A hybrid, Energy-efficient, Distributed Clustering Approach for Adhoc Networks, IEEE Transactions on Mobile Computing, vol. 3, no. 4, Oct.-Dec. 2004, pp. 366-369.

[15] A. Manjeshwar, D.P. Agrawal, TEEN: a protocol for enhanced efficiency in wireless sensor networks, in: Proceedings of the 1st International Workshop on Parallel and Distributed Computing Issues in Wireless Networks and Mobile Computing, San Francisco, CA, April 2001.

[16] A. Manjeshwar, D.P. Agrawal, APTEEN: a hybrid protocol for efficient routing and comprehensive information retrieval in wireless sensor networks, in: Proceedings of the 2nd International Workshop on Parallel and Distributed Computing Issues in Wireless Networks and Mobile computing, Ft. Lauderdale, FL, April 2002.

[17] Y. Xu, J. Heidemann, D. Estrin, Geography-informed energy conservation for ad hoc routing, in: Proceedings of the $7^{\text {th }}$ Annual ACM/IEEE International Conference on Mobile Computing and Networking (MobiCom_01), Rome, Italy, July 2001.
[18] Y. Yu, D. Estrin, R. Govindan, Geographical and energy aware routing: a recursive data dissemination protocol for wireless sensor networks, UCLA Computer Science Department Technical Report, UCLA-CSD TR-01-0023, May 2001.

[19] R. Alasem, A. Reda, and M. Mansour, "Location Based Energy- Efficient Reliable Routing Protocol for Wireless Sensor Networks", Recent Researches in Communications, Automation, Signal processing, Nanotechnology, Astronomy and Nuclear Physics, WSEAS Press, Cambridge, UK, 2011, ISBN: 978-960-474-276-9.

[20] V. Rodoplu, T.H. Ming, Minimum energy mobile wireless networks, IEEE Journal of Selected Areas in Communications 17 (8) (1999) 1333-1344.

[21] L. Li, J. Y Halpern, Minimum energy mobile wireless networks revisited, in: Proceedings of IEEE International Conference on Communications (ICC_01), Helsinki, Finland, June 2001.

[22] Subhadra Shaw (Bose), Energy-Efficient Routing Protocol in Wireless Sensor Network, International Journal of Scientific \& Engineering Research Volume 2, Issue 12, December-2011.

[23] Shamsad Parvin and Muhammad Sajjadur Rahim, Routing Protocols for Wireless Sensor Networks: A Comparative Study, International Conference on Electronics, Computer and Communication (ICECC 2008) University of Rajshahi, Bangladesh.

[24] A Thesis by Gaurav Sharma, Routing In Wireless Sensor Networks, Thapar University, Patiala, India 